\title{
Update on transgenic animal technology
}

\author{
Reviewed by Gregory P. Boivin, DVM
}

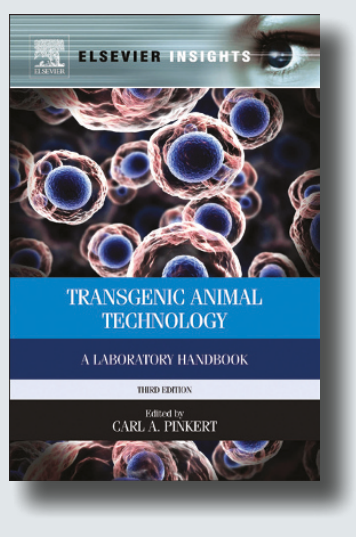

TRANSGENIC ANIMAL

TECHNOLOGY: A LABORATORY

HANDBOOK, 3RD EDN.

Edited by Carl A. Pinkert

Academic, Waltham, MA

Price: $\$ 99.95$

Hardcover, 696 pages

ISBN: 978-0-12-410490-7

The third edition of Transgenic Animal Technology stays true to the book's original intent to provide a solid text describing the technical aspects of genetic manipulation of animals. Carl Pinkert is well known in the field of transgenic technology and has been an integral part of the transgenic world since the 1980s. His knowledge of the transgenic field is a definite advantage as he is very able to detail the importance of transgenic procedures and results. Pinkert has also gathered a group of renowned experts to author the 24 chapters of this book that highlight methods of gene targeting, transferring and editing. A large portion of the text is dedicated to the production of different transgenic animals including fish, birds and mammals, with more than 200 pages describing the many different techniques that have been perfected for transgenic mice. Many of the chapters are similar to the second edition of the text but with updated references and expanded text. Three new chapters include 'Alternative Methods for Transgenesis in Domestic Animal Species', 'Analysis of Phenotyping' and 'Modifying Mitochondrial Genetics'.

The book is very easy to read; it can be a valuable laboratory asset for any researcher working with genetically modified animals, and it can serve as an introductory guide for novices to the field. Each chapter typically provides a historical reference for the methodology it describes, which is a great background introduction to the chapter. Chapters also provide examples of applications for the genetically modified animals that they describe, demonstrating some of the valuable contributions of genetically engineered animals to medical advances. Finally, the methodologies provide good examples of techniques for carrying out a variety of transgenic procedures, and these are well referenced. Descriptions of techniques are thorough and very easy to understand, and they provide step-by-step procedures in good detail.

Two of the new chapters are of particular interest for the laboratory animal community. 'Analysis of Phenotyping' provides a good template for the pathologic analysis of animals based on the requirements of the International Mouse Phenotyping Consortium. This will be invaluable for investigators starting in pathology to ensure that they are collecting the tissues most commonly needed for analysis. 'Modifying Mitochondrial Genetics' addresses a procedure that is not necessarily new but is still rather young in its use. New techniques for mitochondrial manipulation will have a great impact on diseases that previously could not be modeled well, and these techniques are outlined well by the chapter's authors.

One drawback of this book is that it provides very rudimentary figures, often in duplicate and all in black and white. These photographs and figures could have been updated since the second edition of the book to provide a better guidance system for readers. There is also some redundancy across chapters as some techniques are reproduced in multiple chapters.

This is partially attributable to the use of different authors for the different chapters. Another point of weakness is that some chapters have little additional information compared to those of the second edition of the text. This is somewhat disappointing in this rapidly evolving field. However, as many of the referenced techniques have not changed, it may be that the only information in need of updating was the current applications of these techniques to new disease models. Accordingly, there is updated information on the impact of the procedures in most of the chapters.

This text is recommended for those interested in the methodological approaches to transgenic animal production. But for those who already own an earlier edition of Transgenic Animal Technology, this third edition is not a major upgrade and likely should not be recommended as an addition to their libraries. 